\title{
Zinc triflate promoted general synthetic protocol for the facile construction of chromenes and pyrimidines bearing $\mathbf{N}$-allyl quinolone nucleus
}

\author{
Nileshkumar D. Vala ${ }^{1}$, Hardik H. Jardosh ${ }^{2}$, Manish P. Patel ${ }^{1 *}$ \\ ${ }^{1}$ Department of Chemistry, Sardar Patel University, Vallabh Vidyanagar-388120, Gujarat, India \\ ${ }^{2}$ Present address: DSIR laboratory, Cheminova India Ltd., Panoli, Bharuch-394116, Gujarat, India \\ *E-mail address: patelmanish1069@yahoo.com
}

\begin{abstract}
A series of 8 derivatives of chromene 5a-h synthesized via one-pot cyclocondensation reaction of $N$-allyl quinolones, $\alpha / \beta$ naphthol and malononitrile. And 8 derivatives of pyrimidines 8a-h bearing $\mathrm{N}$-allyl quinolone have been synthesized by $N$-allyl quinolones, ethylacetoacetate, thiourea/urea in the presence of zinc triflate as less toxic catalyst. This methodology allowed the high conversion of product at room temperature. Titled derivatives were elucidated by FT-IR, ${ }^{1} \mathrm{H}$ NMR, ${ }^{13} \mathrm{C}$ NMR, elemental analysis, and mass spectral data.
\end{abstract}

Keywords: chromenes; pyrimidines; $N$-ally quinolone; zinc triflate

\section{INTRODUCTION}

Importance of heterocyclic compounds like pyrimidine and chromene are not unknown in the area of medicinal chemistry. From last few decades these compounds are gaining increasingly importance due to their therapeutic and pharmacological properties. Fused chromenes compounds possesses wide spectrum of activities such as antibacterial[1], anticancer agents[2], anti-rhinovirus activity[3], antipyretic, analgesic, anti-inflammatory and antioxidant activities[4]. Pyrimidines are an important class of the heterocyclic compounds possesses various therapeutic and pharmacological properties, like anti-inflammatory and antimicrobial agents[5], anticancer agents[6], antitubercular and antimicrobial activities[7] and antimalarial activity[8].

For the synthesis of pyrimidine derivatives, Biginelli reactions are used which are low yielding condensations reaction and It is catalyzed by various catalyst like, $\mathrm{Mo} / \gamma-\mathrm{Al}_{2} \mathrm{O}_{3}[9]$, $\mathrm{CuS}$ quantum dots[10], Iron(III) tosylate[11], bentonite/PS- $\mathrm{SO}_{3} \mathrm{H}[12]$, N-sulfonic acid poly(4vinylpyridinium) chloride[13] and many more. Most of these catalyst are expensive, harmful and difficult to handle especially on a large scale. Same way various catalyst like, Nano$\mathrm{Fe}_{3} \mathrm{O}_{4}$ encapsulated-silica particles bearing sulfonic acid groups[14], 2hydroxyethylammonium formate[15], sevelamer[16] etc were used for synthesis of chromenes derivative by Knoevenagel condensation followed by Michael addition reaction. These catalyst are also costly, difficult to synthesized and environment effective. In the continuous search of new biologically active compounds[17,18] we targeted to synthesize 
chromene and pyrimidines derivatives. We found that Lewis acid, zinc triflate[19-21] can be used as less toxic catalyst for these reactions, which allows easy work up and absence of side reactions. As shown in table 1 various trial were performed for the optimization of reaction by varying solvent and amount of catalyst, after that we found the best reaction condition which gives high yield at mild reaction conditions. Ethanolic mass of reactants, at room temp and 10 mol\% amount of catalyst, reaction completed within $1.5 \mathrm{~h}$. Further increase in amount of catalyst doesn't give any impact on yield. Portability of solvent to solvent gives much impact on yield of compounds. In that case ethanol founds as a best reaction medium which allows high yield and excellent purity of compounds. This optimized reaction conditions are valid for both chromene and pyrimidines derivatives. All synthesized compounds were characterized by FT-IR, ${ }^{1} \mathrm{H}$ NMR, ${ }^{13} \mathrm{C}$ NMR, elemental analysis, and mass spectral data.

\section{EXPERIMENTAL}

All the reagents and solvents were obtained commercially and used without further purification. All melting points were taken in open capillaries and are uncorrected. For monitoring the progress of all reactions, purity and homogeneity of the synthesized compounds thin-layer chromatography (TLC, on aluminium plates precoated with silicagel, $60 \mathrm{~F}_{254}$, Merck, Darmstadt, Germany) was used; eluent-chloroform: methanol (9:1). UV radiation and/or iodine were used as the visualizing agents. The IR spectra were recorded in $\mathrm{KBr}$ on a Perkin-Elmer Spectrum GX FT-IR Spectrophotometer (Perkin-Elmer, USA) and only the characteristic peaks are reported in $\mathrm{cm}^{-1}$. Elemental analysis $(\% \mathrm{C}, \mathrm{H}, \mathrm{N})$ was carried out by Perkin-Elmer 2400 series-II elemental analyzer (Perkin-Elmer, USA) and all compounds are within $\pm 0.4 \%$ of theory specified. ${ }^{1} \mathrm{H}$ NMR and ${ }^{13} \mathrm{C}$ NMR spectra were recorded in $\mathrm{DMSO}-\mathrm{d}_{6}$ and $\mathrm{CDCl}_{3}$ on a Bruker Avance $400 \mathrm{~F}(\mathrm{MHz})$ spectrometer (Bruker Scientific Corporation Ltd., Switzerland) using TMS as an internal standard at 400 $\mathrm{MHz}$ and $100 \mathrm{MHz}$ respectively. Chemical shifts are reported in parts per million (ppm). Mass spectra were scanned on a Shimadzu LCMS 2010 spectrometer (Shimadzu, Tokyo, Japan)

\subsection{Synthesis of chromene derivatives 5a-h}

A $100 \mathrm{~mL}$ round bottomed flask, was charged with a mixture of 1-allyl-quinolone-3carbaldehydes 3a-d $(3 \mathrm{mmol})$, malononitrile $2(3 \mathrm{mmol})$ and $\beta / \alpha$ naphthol $\mathbf{3} / 4$ (3 mol) in ethanol $(15 \mathrm{~mL})$ containing $\mathrm{Zn}(\mathrm{OTf})_{2}(10 \mathrm{~mol} \%)$. The mixture was allowed to stir at room temperature for $1-1.5 \mathrm{hr}$ and the progress of the reaction was monitored by TLC. After the completion of reaction (as evidenced by TLC), the solid mass separated was collected by filtration, washed well with ethanol-water $(15 \mathrm{~mL})$ and purified by leaching in equal volume ratio of chloroform and methanol $(20 \mathrm{~mL})$ to obtain pure solid sample.

\subsection{Synthesis of pyrimidines derivatives 8a-h}

A $100 \mathrm{~mL}$ round bottomed flask, was charged with a mixture of 1-allyl-quinolone-3carbaldehydes 3a-d $(3 \mathrm{mmol})$, ethylacetoacetate $6(3 \mathrm{mmol})$ and thi/urea $7 \mathrm{a} / \mathrm{b}(3 \mathrm{mmol})$ in ethanol $(15 \mathrm{~mL})$ containing $\mathrm{Zn}(\mathrm{OTf})_{2}(10 \mathrm{~mol} \%)$. The mixture was allowed to stir at room temperature for $1-1.5 \mathrm{hr}$ and the progress of the reaction was monitored by TLC. After the completion of reaction (as evidenced by TLC), the solid mass separated was collected by filtration, washed well with ethanol-water $(15 \mathrm{~mL})$ and recrystalized by absolute ethanol. Then all compounds are subjected for structural analysis. 


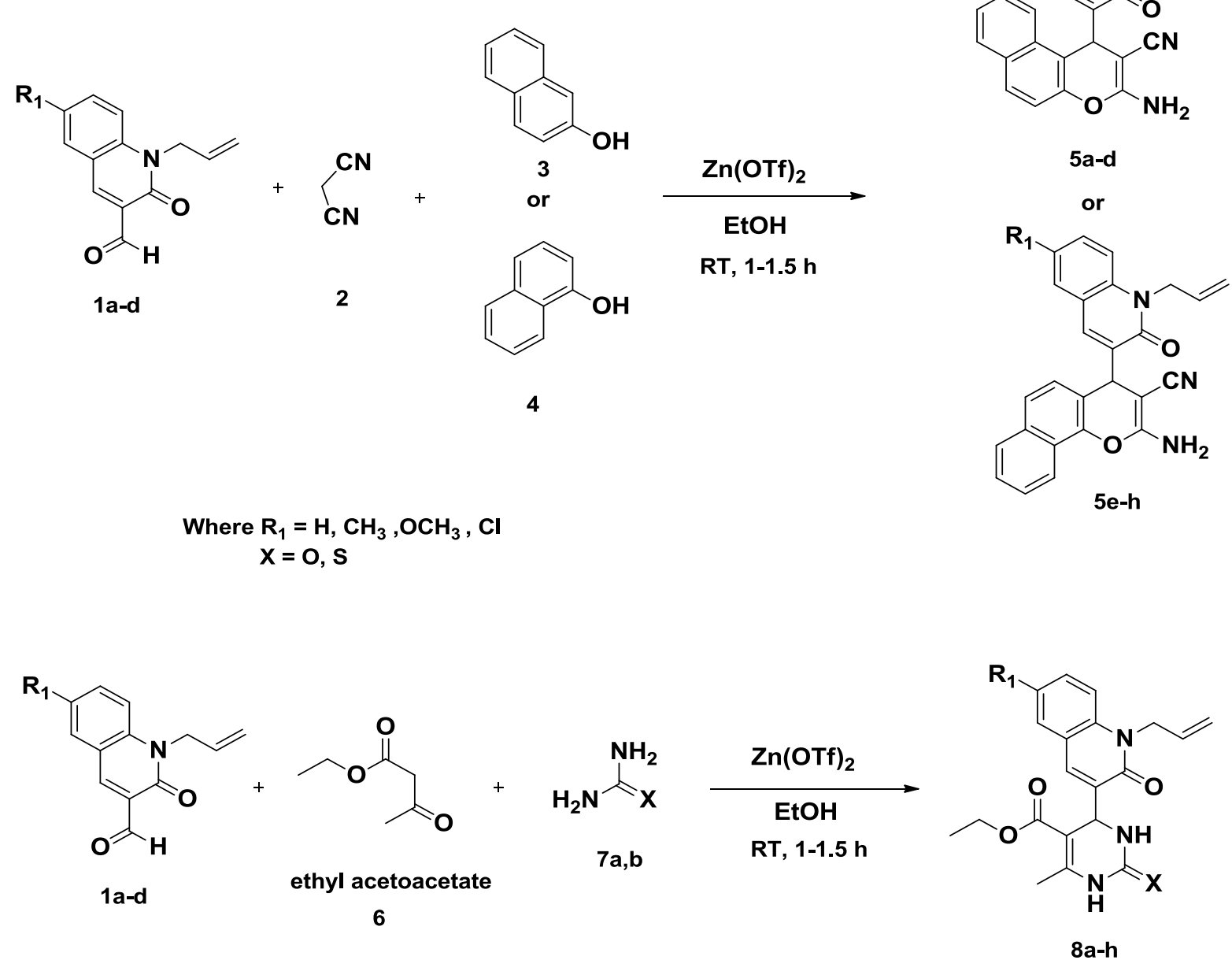

Scheme 1. $\mathrm{Zn}(\mathrm{OTf})_{2}$ promoted synthesis of chromene and pyrimidine derivatives.

Table 1. Optimization of reaction for synthesis of $5 \mathrm{a}$ and $8 \mathrm{a}$.

\begin{tabular}{|c|c|c|c|c|}
\hline Entry & Amount of catalyst (mol\%) & Solvent & \% Yield 5a & \% Yield 8a \\
\hline 1 & 5 & EtOH & 75 & 72 \\
\hline 2 & 8 & EtOH & 81 & 71 \\
\hline $\mathbf{3}$ & $\mathbf{1 0}$ & $\mathbf{E t O H}$ & $\mathbf{8 8}$ & $\mathbf{7 9}$ \\
\hline 4 & 12 & EtOH & 87 & 77 \\
\hline 5 & 10 & $\mathrm{CH}_{3} \mathrm{CN}$ & 62 & 72 \\
\hline 6 & 10 & $\mathrm{Toluene}$ & 58 & 63 \\
\hline 7 & 10 & $\mathrm{CH}_{2} \mathrm{Cl}_{2}$ & 36 & 41 \\
\hline 8 & 10 & $1,4 \mathrm{Dioxane}$ & 33 & - \\
\hline
\end{tabular}

“-” No reaction 

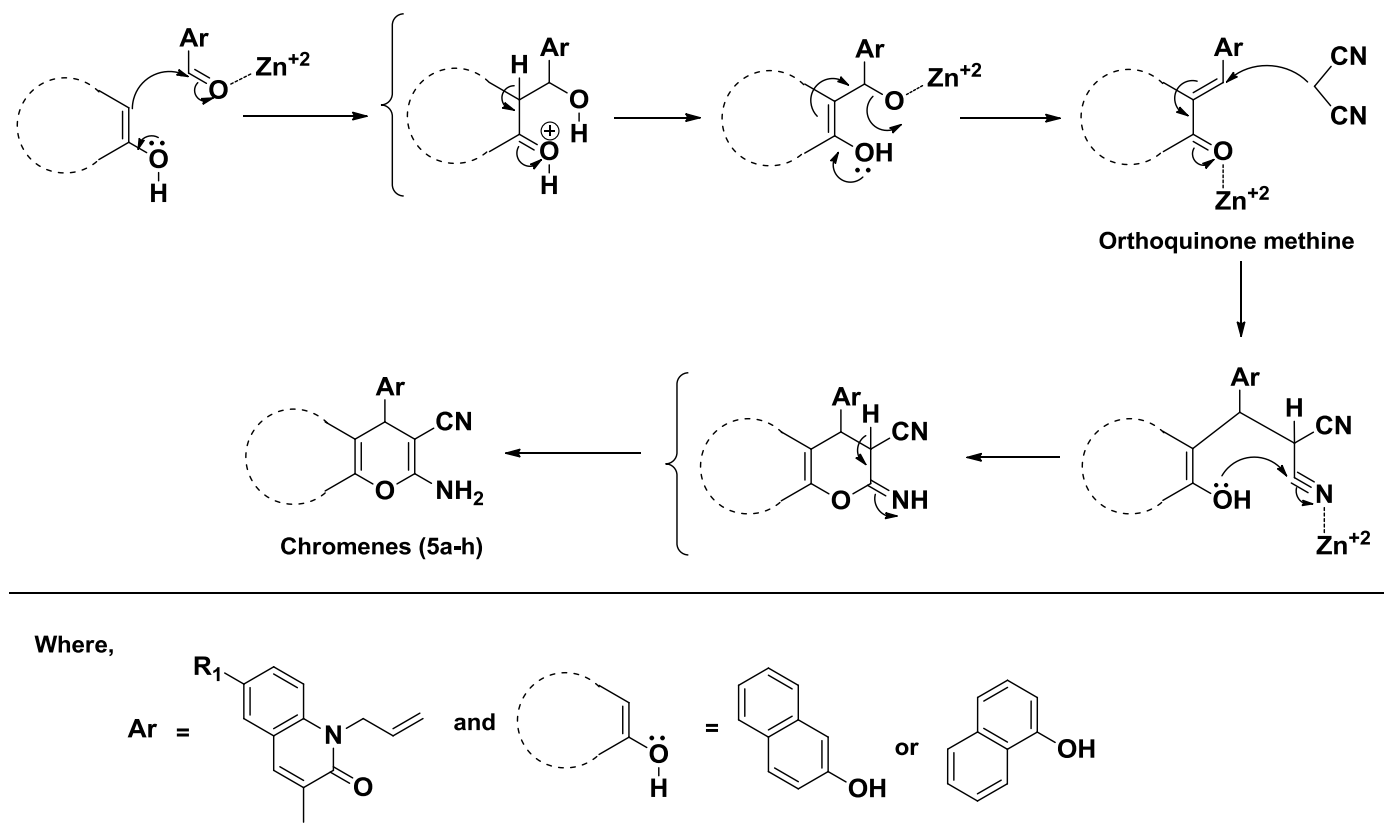
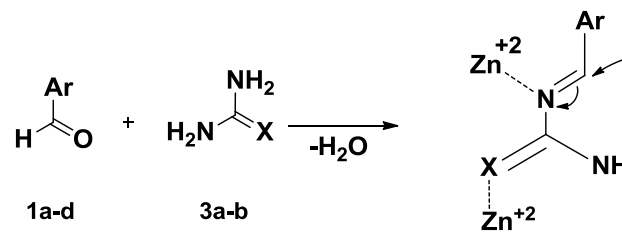

Acyliminium ion Int. 1
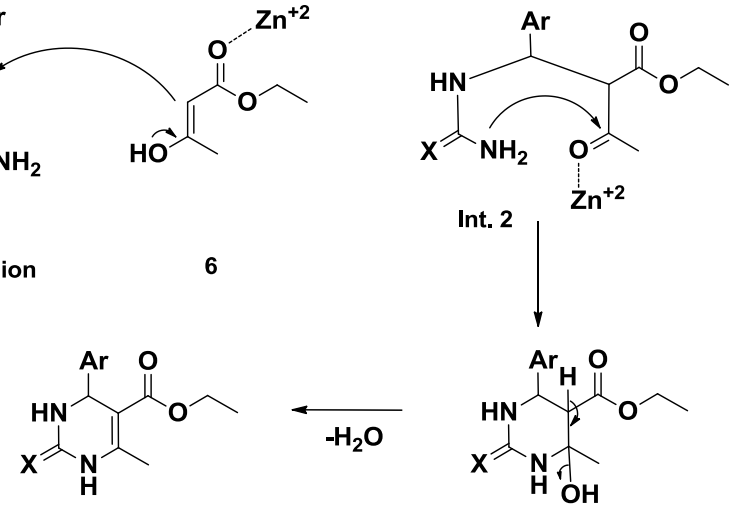

Pyrimidines (8a-h)

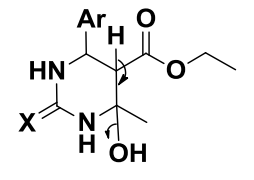

Int. 3

Scheme 2. Plausible mechanism for the formation of chromenes (5a-h) and pyrimidines (8a-h).

The formation of compounds $8 \mathrm{a}-\mathrm{h}$ may proceed via two steps (Scheme 2): i) the formation of arylidene(thio)urea Int-1 and ii) interception of the acyliminium ion intermediate by an activated $\beta$-diketone to produce an open chain urea Int-2, which subsequently undergoes cyclization and dehydration via Int-3 to afford the corresponding pyrimidine derivative. The formation of an acyliminium ion was reported to be the ratedetermining step[22]. $\mathrm{Zn}(\mathrm{OTf})_{2}$ is thought to accelerate the formation of the arylidene(thio)urea Int- 1 and activate the $\beta$-diketone 6 by forming its metal enolate, thus facilitating the addition reaction with a coordinated acyliminium ion.

A general mechanism for the formation of the chromene derivatives $5 \mathrm{a}-\mathrm{h}$ is outlined in scheme 2. The reaction occurs via an in situ initial formation of the orthoquinone methine, containing the electron-poor $\mathrm{C}=\mathrm{C}$ double bond, from the Knoevenagel condensation between activated 1-allyl-quinolone-3-carbaldehydes $1 \mathrm{a}-\mathrm{d}$ and $\beta / \alpha$ naphthol 3/4. Finally, Michael addition of malononitrile to the orthoquinone methane take place, then cyclization leads to formation of chromene derivatives 5a-h. 


\section{SPECTRAL DATA}

\section{1-(1-allyl-2-oxo-1,2-dihydroquinolin-3-yl)-3-amino-1H-benzo[f]chromene-2-carbonitrile} (5a)

Yield: 88\%; mp 198-196 ${ }^{\circ} \mathrm{C}$. IR (KBr) $v_{\max }\left(\mathrm{cm}^{-1}\right)$ : 3023 (Ar str.) 1688, 1647 (C=O str.) 3406 \& 3312 (asym. \& sym. str. of $\left.\mathrm{NH}_{2}\right) 2199(\mathrm{C} \equiv \mathrm{N}), 1251$ (ArC-O-C ether). ${ }^{1} \mathrm{H}$ NMR $\left(\mathrm{DMSO}_{6}\right) \delta_{\mathrm{H}} 4.70-5.23(\mathrm{~m}, 6 \mathrm{H}), 5.81(\mathrm{~s}, 1 \mathrm{H}), 5.93-6.02(\mathrm{~m}, 1 \mathrm{H}), 7.12-7.89(\mathrm{~m}, 11 \mathrm{H}) .{ }^{13} \mathrm{C}$ NMR (DMSO-d ${ }_{6}$ ) 39.87, 42.85, 68.55, 111.13, 112.47, 113.63, 117.4, 121.69, 122.33, 124.68, $126.98,126.99,130.14,130.41,130.45,130.71,130.74,131.70,133.45,133.80,134.42$, 139.89, 145.11, 149.87, 162.19, 166.21. $\mathrm{C}_{26} \mathrm{H}_{19} \mathrm{~N}_{3} \mathrm{O}_{2}(405.45) \mathrm{MS}\left(\mathrm{M}^{+}\right) 406.2$ : calc. C 77.02, H 4.72, N 10.36; found C 77.29, H 4.43, N 10.65\%

1-(1-allyl-6-methyl-2-oxo-1,2-dihydroquinolin-3-yl)-3-amino-1H-benzo[f]chromene-2carbonitrile (5b)

Yield: $73 \%$; mp 186-191 ${ }^{\circ} \mathrm{C}$. IR (KBr) $v_{\max }\left(\mathrm{cm}^{-1}\right)$ : 3016 (Ar str.) 1679, 1653 (C=O str.) 3419 \& 3298 (asym. \& sym. str. of $\left.\mathrm{NH}_{2}\right) 2199(\mathrm{C} \equiv \mathrm{N}), 1243$ (ArC-O-C ether). ${ }^{1} \mathrm{H}$ NMR $\left(\mathrm{DMSO}_{\mathrm{d}}\right) \delta_{\mathrm{H}} 2.18(\mathrm{~s}, 3 \mathrm{H}), 4.78-5.19(\mathrm{~m}, 6 \mathrm{H}), 5.86(\mathrm{~s}, 1 \mathrm{H}), 5.96-6.01(\mathrm{~m}, 1 \mathrm{H}), 7.15-7.86(\mathrm{~m}$, $10 \mathrm{H}){ }^{13} \mathrm{C}$ NMR (DMSO-d 6 ) 18.8, 39.87, 42.85, 68.55, 111.13, 112.08, 112.47, 117.4, 121.14, 122.33, 124.64, 126.99, 127.7, 130.41, 130.46, 130.71, 131.7, 132.9, 133.32, 133.45, 134.23, 134.42, 139.89, 144.17, 149.87, 162.19, 166.21. $\mathrm{C}_{27} \mathrm{H}_{21} \mathrm{~N}_{3} \mathrm{O}_{2}(419.47) \mathrm{MS}\left(\mathrm{M}^{+}\right) 419.65$ : calc. C 77.31, H 5.05, N 10.02; found C 77.25, H 4.95, N 10.15\%

1-(1-allyl-6-methoxy-2-oxo-1,2-dihydroquinolin-3-yl)-3-amino-1H-benzo[f]chromene-2carbonitrile (5c)

Yield: 78\%; mp 175-178 ${ }^{\circ} \mathrm{C}$. IR (KBr) $v_{\max }\left(\mathrm{cm}^{-1}\right)$ : 3020 (Ar str.) 1681, 1652 (C=O str.) $3400 \& 3292$ (asym. \& sym. str. of $\left.\mathrm{NH}_{2}\right) 2210(\mathrm{C} \equiv \mathrm{N}), 1246$ (ArC-O-C ether). ${ }^{1} \mathrm{H}$ NMR $\left(\right.$ DMSO-d $\left._{6}\right) \delta_{\mathrm{H}} 3.74(\mathrm{~s}, 3 \mathrm{H}), 4.83-5.12(\mathrm{~m}, 6 \mathrm{H}), 5.89(\mathrm{~s}, 1 \mathrm{H}), 5.99-6.07(\mathrm{~m}, 1 \mathrm{H}), 6.95-7.82(\mathrm{~m}$, $10 \mathrm{H}) . \mathrm{C}_{27} \mathrm{H}_{21} \mathrm{~N}_{3} \mathrm{O}_{3}$ (435.47) MS ( $\left.\mathrm{M}^{+}\right)$434.87: calc. C 74.47, $\mathrm{H} \mathrm{4.86,} \mathrm{N} \mathrm{9.65;} \mathrm{found} \mathrm{C} 74.66$, $\mathrm{H} 5.07, \mathrm{~N} 9.76 \%$

1-(1-allyl-6-chloro-2-oxo-1,2-dihydroquinolin-3-yl)-3-amino-1H-benzo[f]chromene-2carbonitrile (5d)

Yield: $70 \%$; mp 181-183 ${ }^{\circ} \mathrm{C}$. IR (KBr) $v_{\max }\left(\mathrm{cm}^{-1}\right): 3015$ (Ar str.) 1679, 1646 (C=O str.) 3410 \& 3316 (asym. \& sym. str. of $\left.\mathrm{NH}_{2}\right) 2199(\mathrm{C} \equiv \mathrm{N}), 1254$ (ArC-O-C ether). ${ }^{1} \mathrm{H}$ NMR $\left(\right.$ DMSO-d $\left._{6}\right) \delta_{\mathrm{H}}$ 4.88-5.17 (m, 6H), $5.85(\mathrm{~s}, 1 \mathrm{H})$, 5.96-6.03 $(\mathrm{m}, 1 \mathrm{H}), 7.18-7.92(\mathrm{~m}, 10 \mathrm{H})$. $\mathrm{C}_{26} \mathrm{H}_{18} \mathrm{ClN}_{3} \mathrm{O}_{2}$ (439.89) $\mathrm{MS}\left(\mathrm{M}^{+}\right) 440.12$ : calc. C 70.99, $\mathrm{H} \mathrm{4.12,} \mathrm{N} \mathrm{9.55;} \mathrm{found} \mathrm{C} 70.84, \mathrm{H}$ $4.10, \mathrm{~N} 9.35 \%$

\section{4-(1-allyl-2-oxo-1,2-dihydroquinolin-3-yl)-2-amino-4H-benzo[h] chromene-3-carbonitrile} (5e)

Yield: 79\%; mp 171-174 ${ }^{\circ} \mathrm{C}$. IR (KBr) $v_{\max }\left(\mathrm{cm}^{-1}\right)$ : 3021 (Ar str.) 1681, 1655 (C=O str.) 3418 \& 3296 (asym. \& sym. str. of $\left.\mathrm{NH}_{2}\right) 2206(\mathrm{C} \equiv \mathrm{N}), 1250$ (ArC-O-C ether). ${ }^{1} \mathrm{H}$ NMR $\left(\mathrm{DMSO}_{-} \mathrm{d}_{6}\right) \delta_{\mathrm{H}} 4.89-5.12(\mathrm{~m}, 6 \mathrm{H}), 5.19(\mathrm{~s}, 1 \mathrm{H}), 5.86-5.93(\mathrm{~m}, 1 \mathrm{H}), 7.17-7.25(\mathrm{~m}, 11 \mathrm{H}) .{ }^{13} \mathrm{C}$ 
NMR (DMSO-d $\left.{ }_{6}\right) 39.86,42.85,68.55,111.13,113.63,117.37,117.4,121.69,122.67,122.79$, 124.37, 125.12, 125.13, 126.9, 130.14, 130.45, 130.74, 130.97, 131.7, 132.07, 133.08, 133.8, 145.11, 148.03, 162.19, 166.21. $\mathrm{C}_{26} \mathrm{H}_{19} \mathrm{~N}_{3} \mathrm{O}_{2}$ (405.45) MS $\left(\mathrm{M}^{+}\right)$406.2: calc. C 77.02, H 4.72, N 10.36; found C 76.98, H 4.73, N $10.28 \%$

4-(1-allyl-6-methyl-2-oxo-1,2-dihydroquinolin-3-yl)-2-amino-4H-benzo[h]chromene-3carbonitrile (5f)

Yield: $83 \%$; mp 188-190 ${ }^{\circ} \mathrm{C}$. IR (KBr) $v_{\max }\left(\mathrm{cm}^{-1}\right)$ : 3021 (Ar str.) 1688, 1653 (C=O str.) $3421 \& 3303$ (asym. \& sym. str. of $\left.\mathrm{NH}_{2}\right) 2196(\mathrm{C} \equiv \mathrm{N}), 1244$ (ArC-O-C ether). ${ }^{1} \mathrm{H}$ NMR $\left(\mathrm{DMSO}_{-} \mathrm{d}_{6}\right) \delta_{\mathrm{H}} 2.21(\mathrm{~s}, 3 \mathrm{H}), 4.73-5.01(\mathrm{~m}, 6 \mathrm{H}), 5.23(\mathrm{~s}, 1 \mathrm{H}), 5.88-5.96(\mathrm{~m}, 1 \mathrm{H}), 7.23-7.88(\mathrm{~m}$, $10 \mathrm{H}) . \mathrm{C}_{27} \mathrm{H}_{21} \mathrm{~N}_{3} \mathrm{O}_{2}$ (419.47) MS $\left(\mathrm{M}^{+}\right) 418.96$ : calc. C 77.31, H 5.05, N 10.02; found C 77.58, H 5.33, N 9.84\%

4-(1-allyl-6-methoxy-2-oxo-1,2-dihydroquinolin-3-yl)-2-amino-4H-benzo[h]chromene-3carbonitrile (5g)

Yield: $81 \%$; mp 176-179 ${ }^{\circ} \mathrm{C}$. IR (KBr) $v_{\max }\left(\mathrm{cm}^{-1}\right)$ : 3019 (Ar str.) 1698, 1638 (C=O str.) 3422 \& 3308 (asym. \& sym. str. of $\left.\mathrm{NH}_{2}\right) 2199(\mathrm{C} \equiv \mathrm{N}), 1250$ (ArC-O-C ether). ${ }^{1} \mathrm{H}$ NMR $\left(\mathrm{DMSO}_{-} \mathrm{d}_{6}\right) \delta_{\mathrm{H}} 3.77(\mathrm{~s}, 3 \mathrm{H}), 4.83-5.14(\mathrm{~m}, 6 \mathrm{H}), 5.29(\mathrm{~s}, 1 \mathrm{H}), 5.92-6.02(\mathrm{~m}, 1 \mathrm{H}), 7.12-7.94(\mathrm{~m}$, $10 \mathrm{H}) . \mathrm{C}_{27} \mathrm{H}_{21} \mathrm{~N}_{3} \mathrm{O}_{3}(435.47) \mathrm{MS}\left(\mathrm{M}^{+}\right) 435.16$ : calc. C 74.47, $\mathrm{H}$ 4.86, N 9.65; found $\mathrm{C} 74.69$, $\mathrm{H} 4.75$, N $9.39 \%$

\section{4-(1-allyl-6-chloro-2-oxo-1,2-dihydroquinolin-3-yl)-2-amino-4H-benzo[h]chromene-3- carbonitrile (5h)}

Yield: $74 \%$; $\mathrm{mp} 163-165^{\circ} \mathrm{C}$. IR (KBr) $v_{\max }\left(\mathrm{cm}^{-1}\right)$ : 3033 (Ar str.) 1691, 1649 (C=O str.) 3419 \& 3321 (asym. \& sym. str. of $\left.\mathrm{NH}_{2}\right) 2206(\mathrm{C} \equiv \mathrm{N}), 1255$ (ArC-O-C ether). ${ }^{1} \mathrm{H}$ NMR $\left(\right.$ DMSO-d $\left._{6}\right) \delta_{\mathrm{H}} 4.86-5.16(\mathrm{~m}, 6 \mathrm{H}), 5.31(\mathrm{~s}, 1 \mathrm{H}), 5.90-5.98(\mathrm{~m}, 1 \mathrm{H}), 7.26-7.96(\mathrm{~m}, 10 \mathrm{H})$. $\mathrm{C}_{26} \mathrm{H}_{18} \mathrm{ClN}_{3} \mathrm{O}_{2}$ (439.89) MS $\left(\mathrm{M}^{+}\right) 440.14$ : calc. C 70.99, $\mathrm{H} \mathrm{4.12,} \mathrm{N} \mathrm{9.55;} \mathrm{found} \mathrm{C} 71.12, \mathrm{H}$ $3.86, \mathrm{~N} 9.34 \%$

Ethyl 4-(1-allyl-2-oxo-1,2-dihydroquinolin-3-yl)-6-methyl-2-oxo-1,2,3,4tetrahydropyrimidine-5-carboxylate (8a)

Yield: 79\%; mp 190-193 ${ }^{\circ} \mathrm{C}$. IR (KBr) v maz: 3020 (Ar str.) 3397,3219, 3108 (N-H Str), 1706, 1658 (C=O Str.) 1224 (C-O Str.). ${ }^{1} \mathrm{H}$ NMR (DMSO-d $\left.{ }_{6}\right) \delta_{\mathrm{H}} 1.01-1.05$ (t, 3H), 2.36 (s, $3 \mathrm{H}), 3.94-3.99$ (q, $2 \mathrm{H}), 4.88-5.16(\mathrm{~m}, 4 \mathrm{H}), 5.44(\mathrm{~s}, 1 \mathrm{H}), 5.90-5.97(\mathrm{~m}, 1 \mathrm{H}), 7.15-7.78(\mathrm{~m}$, $6 \mathrm{H}), 9.23(\mathrm{~s}, 1 \mathrm{H}) .{ }^{13} \mathrm{C}$ NMR $\left(\mathrm{DMSO}_{-} \mathrm{d}_{6}\right)$ 14.55, 18.39, 44.39, 50.62, 59.52, 96.54, 115.32, $117.02,120.17,122.58,129.57,130.93,132.98,133.13,135.07,138.66,150.68,152.56$, 160.70, 165.66. $\mathrm{C}_{20} \mathrm{H}_{21} \mathrm{~N}_{3} \mathrm{O}_{4}(367.40) \mathrm{MS}\left(\mathrm{M}^{+}\right)$366.81: calc. C 65.38, H 5.76, N 11.44; found C 65.48, H 5.79, N $11.27 \%$

Ethyl 4-(1-allyl-6-methyl-2-oxo-1,2-dihydroquinolin-3-yl)-6-methyl-2-oxo-1,2,3,4tetrahydropyrimidine-5-carboxylate $(8 \mathrm{~b})$

Yield: $68 \%$; mp 187-189 ${ }^{\circ} \mathrm{C}$. IR (KBr) $v_{\max }\left(\mathrm{cm}^{-1}\right): 3020$ (Ar str.) 3397,3219, $3103(\mathrm{~N}-\mathrm{H}$ Str), 1706, 1658 (C=O Str.) 1224 (C-O Str.). ${ }^{1} \mathrm{H}$ NMR (DMSO-d $\left.{ }_{6}\right) \delta_{\mathrm{H}} 1.06-1.09$ (t, 3H), 2.26 
(s, 3H), $2.39(\mathrm{~s}, 3 \mathrm{H}), 3.91-4.05(\mathrm{q}, 2 \mathrm{H}), 4.78-4.98(\mathrm{~m}, 4 \mathrm{H}), 5.33(\mathrm{~s}, 1 \mathrm{H}), 5.79-5.88(\mathrm{~m}, 1 \mathrm{H})$, 7.21-7.82 (m, 5H), $9.18(\mathrm{~s}, 1 \mathrm{H}) . \mathrm{C}_{21} \mathrm{H}_{23} \mathrm{~N}_{3} \mathrm{O}_{4}(381.43) \mathrm{MS}\left(\mathrm{M}^{+}\right)$381.35: calc. C 66.13, $\mathrm{H}$ 6.08, N 11.02; found C 65.96, H 6.17, N 11.29\%

Ethyl 4-(1-allyl-6-methoxy-2-oxo-1,2-dihydroquinolin-3-yl)-6-methyl-2-oxo-1,2,3,4tetrahydropyrimidine-5-carboxylate $(8 \mathrm{c})$

Yield: $76 \%$; mp 173-176 ${ }^{\circ} \mathrm{C}$. IR (KBr) $v_{\max }\left(\mathrm{cm}^{-1}\right): 3017$ (Ar str.) 3391,3218, $3089(\mathrm{~N}-\mathrm{H}$ Str), 1696, 1656 (C=O Str.) 1218 (C-O Str.). ${ }^{1} \mathrm{H}$ NMR (DMSO-d 6 ) $\delta_{\mathrm{H}} 1.10-1.14$ (t, 3H), 2.42 $(\mathrm{s}, 3 \mathrm{H}), 3.75(\mathrm{~s}, 3 \mathrm{H}), 3.89-3.96(\mathrm{q}, 2 \mathrm{H}), 4.82-5.12(\mathrm{~m}, 4 \mathrm{H}), 5.35(\mathrm{~s}, 1 \mathrm{H}), 5.82-5.89(\mathrm{~m}, 1 \mathrm{H})$, 7.18-7.86 (m, 5H), $9.21(\mathrm{~s}, 1 \mathrm{H}) . \mathrm{C}_{21} \mathrm{H}_{23} \mathrm{~N}_{3} \mathrm{O}_{5}(397.42) \mathrm{MS}\left(\mathrm{M}^{+}\right) 396.92$ : calc. C 63.46, H 5.83, N 10.57; found C 63.55, H 6.04, N 10.78\%

Ethyl 4-(1-allyl-6-chloro-2-oxo-1,2-dihydroquinolin-3-yl)-6-methyl-2-oxo-1,2,3,4tetrahydropyrimidine-5-carboxylate $(8 \mathrm{~d})$

Yield: $69 \%$; mp 168-170 ${ }^{\circ} \mathrm{C}$. IR (KBr) $v_{\max }\left(\mathrm{cm}^{-1}\right)$ : 3029 (Ar str.) 3406, 3223, 3109 (N-H Str), 1700, 1661 (C=O Str.) $1222\left(\mathrm{C}-\mathrm{O}\right.$ Str.). ${ }^{1} \mathrm{H}$ NMR (DMSO-d $\left.{ }_{6}\right) \delta_{\mathrm{H}} 1.12-1.15(\mathrm{t}, 3 \mathrm{H}), 2.43$ (s, 3H), 3.93-4.02 (q, 2H), 4.88-5.22 (m, 4H), $5.39(\mathrm{~s}, 1 \mathrm{H})$, 5.83-5.91 (m, 1H), 7.24-7.93 (m, $5 \mathrm{H}), 9.21(\mathrm{~s}, 1 \mathrm{H}) . \mathrm{C}_{20} \mathrm{H}_{20} \mathrm{ClN}_{3} \mathrm{O}_{4}(401.84) \mathrm{MS}\left(\mathrm{M}^{+}\right)$401.98: calc. C 59.78, H 5.02, N 10.46; found $\mathrm{C} 60.03, \mathrm{H} 4.84, \mathrm{~N} 10.37 \%$

Ethyl 4-(1-allyl-2-oxo-1,2-dihydroquinolin-3-yl)-6-methyl-2-thioxo-1,2,3,4tetrahydropyrimidine-5-carboxylate (8e)

Yield: 73\%; mp 188-190 ${ }^{\circ} \mathrm{C}$. IR (KBr) $v_{\max }\left(\mathrm{cm}^{-1}\right)$ : 3020 (Ar str.) 3399, 3215, $3102(\mathrm{~N}-\mathrm{H}$ Str), 1706, $1653\left(\mathrm{C}=\mathrm{O}\right.$ Str.) $1195\left(\mathrm{C}=\mathrm{S}\right.$ Str.). ${ }^{1} \mathrm{H}$ NMR $\left(\mathrm{DMSO}-\mathrm{d}_{6}\right) \delta_{\mathrm{H}} 1.02-1.05(\mathrm{t}, 3 \mathrm{H}), 2.35$ $(\mathrm{s}, 3 \mathrm{H}), 3.94-4.02(\mathrm{q}, 2 \mathrm{H}), 4.95-5.15(\mathrm{~m}, 4 \mathrm{H}), 5.29(\mathrm{~d}, 1 \mathrm{H}), 5.78-5.85(\mathrm{~m}, 1 \mathrm{H}), 7.06-7.74(\mathrm{~m}$, $5 \mathrm{H}), 8.92(\mathrm{~s}, 1 \mathrm{H}), 10.25(\mathrm{~s}, 1 \mathrm{H}), \mathrm{C}_{20} \mathrm{H}_{21} \mathrm{~N}_{3} \mathrm{O}_{3} \mathrm{~S}(383.46) \mathrm{MS}\left(\mathrm{M}^{+}\right)$382.67: calc. C 62.64, $\mathrm{H}$ 5.52, N 10.96; found C 62.31, H 5.90, N 11.27\%

Ethyl 4-(1-allyl-6-methyl-2-oxo-1,2-dihydroquinolin-3-yl)-6-methyl-2-thioxo-1,2,3,4tetrahydropyrimidine-5-carboxylate (8f)

Yield: $66 \%$; mp 168-171 ${ }^{\circ} \mathrm{C}$. IR (KBr) $v_{\max }\left(\mathrm{cm}^{-1}\right)$ : 3028 (Ar str.) 3401, 3208, $3110(\mathrm{~N}-\mathrm{H}$ Str), 1712, 1655 (C=O Str.) $1195\left(\mathrm{C}=\mathrm{S}\right.$ Str.) ${ }^{1} \mathrm{H}$ NMR (DMSO-d $\left.{ }_{6}\right) \delta_{\mathrm{H}} 1.02-1.06(\mathrm{t}, 3 \mathrm{H}), 2.24$ (s, 3H), $2.34(\mathrm{~s}, 3 \mathrm{H}), 3.95-4.06(\mathrm{q}, 2 \mathrm{H}), 4.78-5.08(\mathrm{~m}, 4 \mathrm{H}), 5.36(\mathrm{~s}, 1 \mathrm{H}), 5.79-5.86(\mathrm{~m}, 1 \mathrm{H})$, 7.04-7.73 (m, 5H), $8.97(\mathrm{~s}, 1 \mathrm{H}), 10.23(\mathrm{~s}, 1 \mathrm{H}) . \mathrm{C}_{21} \mathrm{H}_{23} \mathrm{~N}_{3} \mathrm{O}_{3} \mathrm{~S}$ (397.49) MS (M ${ }^{+}$) 398.01: calc. C 63.45, H 5.83, N 10.57; found C 63.39, H 5.82, N 10.71\%

Ethyl 4-(1-allyl-6-methoxy-2-oxo-1,2-dihydroquinolin-3-yl)-6-methyl-2-thioxo-1,2,3,4tetrahydropyrimidine-5-carboxylate $(8 \mathrm{~g})$

Yield: 76\%; mp 166-168 ${ }^{\circ} \mathrm{C}$. IR (KBr) $\vee_{\text {maz: }} 3016$ (Ar str.) 3396, 3221, 3106 (N-H Str), 1710, 1655 (C=O Str.) $1196\left(\mathrm{C}=\mathrm{S}\right.$ Str.). ${ }^{1} \mathrm{H}$ NMR (DMSO-d $\left.{ }_{6}\right) \delta_{\mathrm{H}} 1.04-1.06(\mathrm{t}, 3 \mathrm{H}), 3.78(\mathrm{~s}$, $3 \mathrm{H}), 2.38(\mathrm{~s}, 3 \mathrm{H}), 3.98-4.08(\mathrm{q}, 2 \mathrm{H}), 4.81-5.10(\mathrm{~m}, 4 \mathrm{H}), 5.34(\mathrm{~s}, 1 \mathrm{H}), 5.81-5.87(\mathrm{~m}, 1 \mathrm{H}), 7.12-$ $7.86(\mathrm{~m}, 5 \mathrm{H}), 9.03(\mathrm{~s}, 1 \mathrm{H}), 10.31(\mathrm{~s}, 1 \mathrm{H}) . \mathrm{C}_{21} \mathrm{H}_{23} \mathrm{~N}_{3} \mathrm{O}_{4} \mathrm{~S}(413.49) \mathrm{MS}\left(\mathrm{M}^{+}\right)$413.28: calc. $\mathrm{C}$ 61.00, H 5.61, N 10.16; found C 60.86, H 5.39, N 10.48\% 


\section{Ethyl 4-(1-allyl-6-chloro-2-oxo-1,2-dihydroquinolin-3-yl)-6-methyl-2-thioxo-1,2,3,4- tetrahydropyrimidine-5-carboxylate $(8 \mathrm{~h})$}

Yield: 64\%; mp 162-165 ${ }^{\circ} \mathrm{C}$. IR (KBr) $v_{\max }\left(\mathrm{cm}^{-1}\right)$ : 3020 (Ar str.) 3403, 3218, 3096 (N-H Str), 1698, 1650 (C=O Str.) $1204\left(\mathrm{C}=\mathrm{S}\right.$ Str.). ${ }^{1} \mathrm{H}$ NMR (DMSO-d $\left.{ }_{6}\right) \delta_{\mathrm{H}} 1.07-1.09(\mathrm{t}, 3 \mathrm{H}), 2.36$ $(\mathrm{s}, 3 \mathrm{H}), 3.96-4.05(\mathrm{q}, 2 \mathrm{H}), 4.89-5.19(\mathrm{~m}, 4 \mathrm{H}), 5.38(\mathrm{~s}, 1 \mathrm{H}), 5.84-5.91(\mathrm{~m}, 1 \mathrm{H}), 7.15-7.91(\mathrm{~m}$, $5 \mathrm{H}), 9.06(\mathrm{~s}, 1 \mathrm{H}), 10.29(\mathrm{~s}, 1 \mathrm{H}) . \mathrm{C}_{20} \mathrm{H}_{20} \mathrm{ClN}_{3} \mathrm{O}_{3} \mathrm{~S}$ (417.91) MS (M $\left.{ }^{+}\right)$416.74: calc. C 57.48, H 4.82, N 10.05; found C 57.21, H 4.96, N 10.21\%

\section{CONCLUSION}

After performing various trial reaction for the synthesis of chromene and pyrimidine derivatives, we found that Zinc triflate catalyst at its $10 \mathrm{~mol} \%$ amount and ethanol as reaction solvent at room temp can be adopted as the best condition. This protocol is applicable for both above mention moiety's derivative. This reaction pathway followed by green approach of less environmental pollution and mild reaction conditions.

\section{Acknowledgement}

The authors are thankful to Professor \& Head, Department of Chemistry, Sardar Patel University for providing ${ }^{1} \mathrm{H}$ NMR spectroscopy, FT-IR and research facilities. We are also thankful to Oxygen healthcare P.Ltd, Ahmedabad for mass spectrometry facilities, SICART, Vallabh Vidya nagar, for elemental. One of the authors (Nileshkumar D. Vala) is grateful to UGC, New Delhi for a BSR Research Fellowship.

\section{References}

[1] Kidwaia M., Poddara R., Bhardwaja S, et al., Eur. J. Med. Chem., 45 (2010) 5031-5038.

[2] Patil A., Wang J., Li X., et al., Bioorg. Med. Chem. Lett., 22 (2012) 4458-4461.

[3] Conti C., Monaco L., Desideri N., Bioorg. Med. Chem., 22 (2014) 1201-1207.

[4] Johnson A., Kumar A., Rasheed S., et al., J. Ethnopharmacology, 130 (2010) 267-271.

[5] Aggarwal R., Masan E., Kaushik P, et al., J. Fluor. Chem., 168 (2014) 16-24.

[6] Shao K., Zhang X., Chen P, et al., Bioorg. Med. Chem Lett, 24 (2014) 3877-3881.

[7] Desai N., Kotadiya G., Bioorg. Med. Chem. Lett, 24 (2014) 3126-3130.

[8] Pretorius I., Breytenbach W., Kock C., et al., Bioorg. Med. Chem., 21 (2013) 269-277.

[9] K. Kouachi, G. Lafaye, S. Pronier, L. Bennini, S. Menad, J. Mol. Catal. A: Chem., 395 (2014) 210-216.

[10] Chaudhary G., Bansal P., Mehta S., Chem. Engineering J., 243 (2014) 217-224.

[11] Starcevich J., Laughlin T., Mohan R., Tet. Lett., 54 (2013) 983-985.

[12] Kalbasi R., Massah A., Daneshvarnejad B., Applied Clay Science, 55 (2012) 1-9.

[13] Shirini F., Abedini M., Pourhasan-Kisomi R., Chin. Chem. Lett., 25 (2014) 111-114. 
[14] Nemati F., Heravi M., Rad R., Chin. J. Catalysis, 33 (2012) 1825-1831.

[15] Shaterian H., Arman M., Rigi F., J. Mol. Liq., 158 (2011) 145-150.

[16] Zhao X., Yang K., Zhang Y., Chin. Chem. Lett., 25 (2014) 1141-1144.

[17] Patel N., Patel R., and Patel M., J. Environ. Res. D. 3 (2009) 851.

[18] Jardosh H, Patel M., Eur. J. Med. Chem. 65 (2013)348-359.

[19] Lekhok K., Bhuyan D., Prajapati D., Mol. Divers, 14 841-846.

[20] Tsengy M., Chu Y., Tetrahedron, 64 (2008) 9515-9520.

[21] Kumar U., Reddy S., Reddy P., et al, Tet. Lett. 55 (2014) 910-912.

[22] Suzuki I., Suzumura Y., Takeda K., Tet. Lett. 47 (2006) 7861-7864. 